\title{
Magnetic Field Dependence of Gate Voltage and Current in a GaAs-Heterostructure in the Quantum Hall Regime
}

\author{
D. Weiss*, V. MOSSeR*, V. Gudmundsson, R.R. Gerhardts, K. V. Klitzing
}

\begin{abstract}
Max-Planck-Institut für Festkörperforschung, Heisenbergstr. 1, D-7000 Stuttgart 80, FRG *Physik-Department TU München, D-8046 Garching
\end{abstract}

(Received Jan. 13,1987 by M. Cardona)

\begin{abstract}
The current flow at a fixed gate voltage and the floating gate voltage for fixed charge density in a gated GaAs heterostructure have been measured as a function of the magnetic field. The voltage oscillations which reflect the behaviour of the chemical potential have been clearly resolved. The experimental results are explained by a statistical model of inhomogeneities in the carrier concentration implying an effective density of states between the Landau levels.
\end{abstract}

Recently, measurements have been performed on GaAs-heterostructures in order to obtain information about the density of states (DOS) of a twodimensional electron gas (2DEG) in a strong quantizing magnetic field. The activation energy of $\rho_{x x}$ [1-3], the capacitance [4-6], the specific heat [7] and the magnetization [8] have been investigated. Some of these measurements are sensitive only in certain energy regions of the DOS. The capacitance measurement fails $[4,5]$ for example in the interesting region between the Landau levels due to the vanishing conductivity of the 2DEG in that region. This problem of high series resistance can be reduced by using a backside contact $\left(n^{+}\right)$instead of the channel contacts [6]. In the present paper we will discuss another type of measurement which is closely related to the capacitance measurement but more sensitive to the actual form of the DOS in the whole magnetic field region, even though channel contacts are used. In this measurement the current between the top gate and the 2DEG is measured as a function of the magnetic field B. This current, the "gate current", is directly related to the changes in the DOS and the capacitance as a function of $\mathbf{B}[2,9]$. We also report a measurement of the gate voltage $V_{g}$, i.e. the voltage between the gate and the $2 \mathrm{DEG}$, as a function of $B$. In comparision to earlier experiments [10] we see clear oscillations which follow quantitatively the variation of the chemical potential $\mu$ of the 2 DEG. The results from the gate current measurement [2] can only be explained poorly by the adhoc simple background model assuming a constant background. On the other hand a recently proposed model $[9,11]$ on statistical inhomogeneities of the carrier density $n_{s}$ is shown to reproduce the experimental results excellently, both in the case of the gate current and the gate voltage. After a description of the experimental set up the inhomogeneity model will briefly be introduced. Then the experimental results will be discussed and compared with calculations based on the inhomogeneity model.
The experiments described here have been carried out on an AlGaAs-GaAs heterostructure with a low temperature mobility $\mu=585,000 \mathrm{~cm}^{2} / V s$ and a carrier density $n_{s}=1.90 \cdot 10^{11} \mathrm{~cm}^{-2}$. The sample has the usual mesa Hall geometry (see Fig. 1) with an Au-gate on top (area $5.65 \mathrm{~mm}^{2}$ ). All potential probes were short circuited and acted as a channel contact. The top layers (GaAs/Si-doped AlGaAs and undoped AlGaAs spacer) are completely depleted and therefore insulating. Both, current (gate voltage $V_{g}=$ const) and voltage (carrier density $n_{s}=$ const) between gate and channel contacts have been measured as a function of the magnetic field using an electrometer (Keithley model 617). In the volts function, the electrometer circuit is set up as a high impedance $\left(>2 \cdot 10^{14} \Omega\right)$, unity gain, noninverting amplifier. In the volts mode guarding was applied. This means, that the high impedance lead is surrounded by a shield, which is kept (from a low impedance source) on the same potential as the signal itself. One advantage of guarding is a reduction

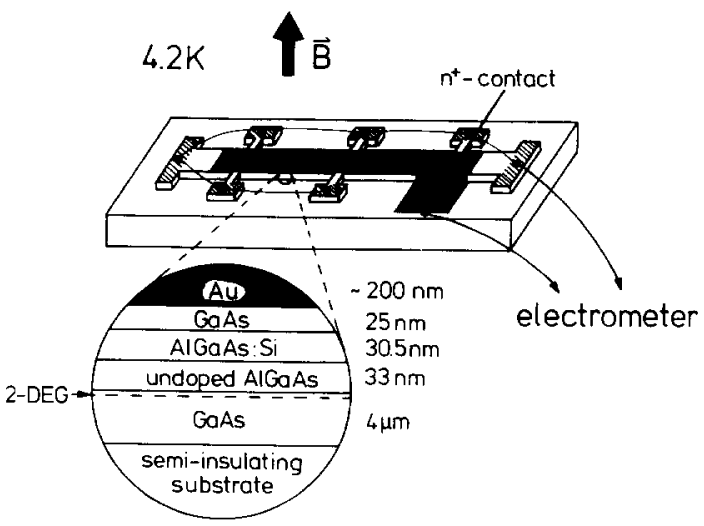

Fig. 1

Schematic experimental set up and structure of the investigated sample 
of the effects of leakage resistance, another is the lowering of the effective input capacitance which reduces the rise time of a measurement. A problem during volts measurements often was the drift of the signal, probably due to the loading of the sample by electrometer leakage currents. This effect is reduced by increasing the sample capacitance. In the amps mode, the circuit is configured as a feedback type current to voltage converter. Three sweeprates of the magnetic field - $2 \mathrm{~T} / \mathrm{min}, 1 \mathrm{~T} / \mathrm{min}$ and $0.5 \mathrm{~T} / \mathrm{min}$ - have been used. The measured voltage signal is within the uncertainty due to the drift of the signal independent of these sweep rates, indicating that the time constants within the experimental set up do not play an important role. The current signal is directly proportional to the sweeprate. The ratio of the sweep rates is exactly reflected in the current signal except for the highest sweep rate $(2 T / \mathrm{min})$ where the signal is somewhat $(\approx 10 \%)$ too low, indicating the finite rise time of the current signal. Changing the sweep direction results in a change of the sign of the current signal contrary to the voltage signal which remains unchanged.

The gate current can be evaluated by solving selfconsistently the Schrödinger and the Poisson equations for the fully 3D heterostructure. The gate voltage $V_{g}$ is related to the variation of the electrostatic potential between the gate and the 2DEG and the electrochemical potential $\mu$ measured with respect to the electric subband energy $E_{0}$ and can be written as [9]

$$
e V_{g}=\mu+\left(E_{0}-E_{s}\right)+\frac{4 \pi e^{2} L}{\kappa_{D}} n_{s}+K
$$

Here $E_{s}$ is the bottom of the potential well at the interface, $\mathrm{L}$ and $\kappa_{D}$ are the thickness and the dielectric constant of the AlGaAs barrier and $\mathrm{K}$ is a constant dependening e.g. on the band offset at the interface and the Schottky barrier at the gate. Whereas the energy eigenvalue $E_{0}-E_{s}$ depends only on the selfconsistently determined electron density $n$, (and the depletion charge density), $\mu$ depends for a given analytical form of the DOS on both $n_{s}$ and the magnetic field $B$, so that the relation

$$
\left(\frac{\partial \mu}{\partial B}\right)_{n_{s}}=-\left(\frac{\partial n_{s}}{\partial B}\right)_{\mu} /\left(\frac{\partial n_{s}}{\partial \mu}\right)_{B}
$$

holds. Keeping the gate voltage $V_{g}$ fixed we obtain for the gate current per unit area

(3) $I_{g}=-e\left(\frac{d n_{s}}{d B}\right)_{V_{g}} \frac{d B}{d t}=-e\left(\frac{\partial n_{s}}{\partial B}\right)_{\mu} \frac{C}{e^{2} D_{T}} \cdot \frac{d B}{d t}$

where the differential capacitance per unit area $C$ and the thermodynamic DOS (TDOS) $D_{T}$ are given by

$$
\frac{1}{C}=\frac{1}{e}\left(\frac{\partial V_{g}}{\partial n_{s}}\right)_{B}, \quad D_{T}=\left(\frac{\partial n_{s}}{\partial \mu}\right)_{B}
$$

It is therefore clear that the current between the gate and the 2DEG is due to the selfconsistent rearrangement of $\mu, n_{s}, \mathrm{C}$ and $D_{T}$ as the magnetic field $\mathrm{B}$ is varied while $V_{g}$ is kept constant (Amperemeter between channel and gate contacts). The gate voltage measurement probes, on the other hand, the oscillations of $\mu$ as $n$, is kept constant (high impedance voltmeter between channel and gate), since under these conditions [9]

$$
e V_{g}=\mu+\text { const } \text {. }
$$

The selfconsistent Born approximation [12] for the scattering of electrons by short range impurities predicts that the DOS between Landau levels (LLs) should vanish in a strong magnetic field. This is in contradiction to experimental results [1-8]. To compare with experiment we shall employ a recent statistical model of inhomogeneities in the electron density $n_{\bullet}[9]$, which has been found to explain well the results of the earlier mentioned indirect measurements of the DOS $[1-9,11]$.

In this model "the $n_{s o}-$ Gaussian model" [9] the density $n_{\text {so }}$ at $\mathrm{B}=0$ is assumed to be Gaussian distributed with a certain standard $\delta n_{\text {,o }}$ which will be chosen for a good agreement between the model and the experiment. The initial DOS fed into the model will be a Gaussian DOS as predicted by the LOCA (lowest order cumulant approximation ) [13] with the level-broadening determined from the mobility of the 2DEG [12]. Hence, only one parameter $\delta n_{\text {so }}$ will be free for fitting. In the case of the gate voltage measurement the electron number density $n_{\text {s }}$ is kept constant so the $n_{\text {so }}$ model reduces to the " $n_{\text {o }}-$ Gaussian model". Measurement and theory of the magnetocapacitance have been published previously $[2,9]$.

Experimental results, obtained from one and the same sample are shown in Fig. 2 where the measured gate voltage $V_{g}$ (a), gate current (b) and capacitance (c) as a function of the magnetic field $B$ are compared with corresponding model calculations with and without introducing inhomogeneities. The calculations are based on a Gaussian model DOS, neglecting spin splitting. The model calculations in Fig. 2 show that there is little difference between the homogeneous and the inhomogeneous case as long as the electrochemical potential $\mu$ is located within a LL and the experimental curves (a)-(c) are well described in this situation by the homogeneous model with $\Gamma=0.18 \sqrt{B[T]} m e V$. If the Fermi level however jumps from one LL to another LL (integer filling factors i) the experimental data no longer can be explained assuming a homogeneous sample with the small linewidth mentioned above. In this case the calculated current maxima are narrower and much too high compared to the experiment (Fig. $\overline{2}$ b). Magnetocapacitance minima (Fig. 2c) show a similar behaviour as long as the capacitance signal is not influenced by the low conductivity state $\left(\sigma_{x x} \rightarrow 0\right)$ of 

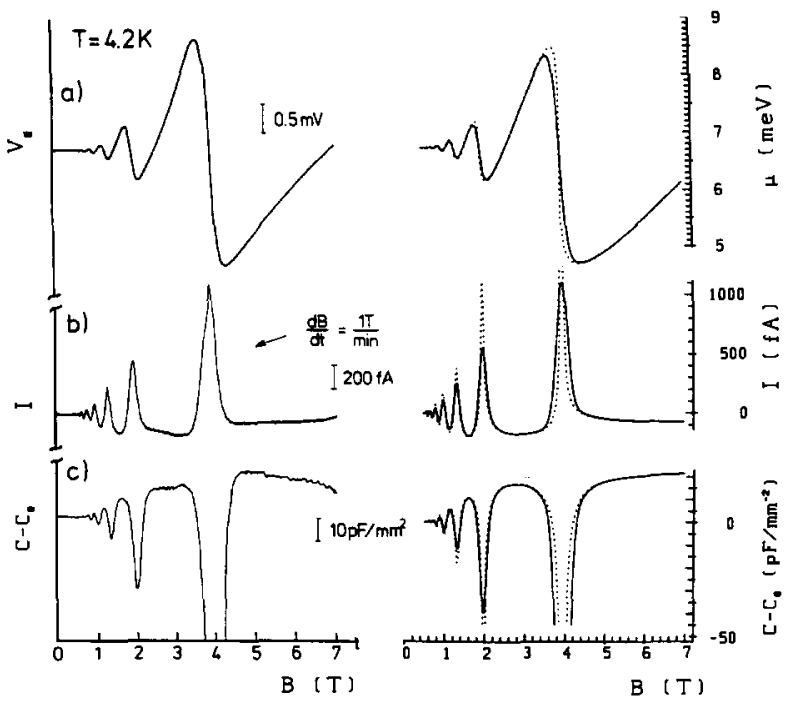

Fig. 2

Comparison of experiment and model calculations for oscillations of the gate voltage $V_{g}$ (a), gate current $I_{g}$ (b) and capacitance (c) as a function of magnetic field B. The calculated curves for the homogeneous case (dashed line) and for the inhomogeneous case (solid line) are based on a Gaussian model DOS with a linewidth $\Gamma=0.18 \sqrt{B[T]} m e V\left(=\Gamma_{S C B A}\right)$. the channel [2]: the calculated magnetocapacitance minima are too deep compared to the experiment (note that the depth and width of the measured capacitance signal for filling factor $i=2$ (at $B \approx 4 T$ ) is mainly governed by the $\sigma_{x x}$-minimum). The oscillations of the gate voltage (Fig. 2a) - directly related to the electrochemical potential $\mu$ via (5) - show somewhat smoother transitions from one LL to another than expected from the homogeneous model.

All these differences between the homogeneous model and the experiment may be explained by introducing artificially a magnetic field dependent background DOS [14]. The presence of this apparent background DOS can be explained by the statistical model of inhomogeneities in the carrier density $n_{\text {s. }}$. The amount of inhomogeneity needed $(3.2 \%)$ is modest and within the experimentally expected limits. The overall shape of the measured curves is well reproduced by the inhomogeneity model (solid lines in Fig. 2). The deviations from the model calculations observed for a Fermi level position within a LL (asymmetric lineshape) are due to the neglect of spin splitting.

We have presented a measurement of the chemical potential in an AlGaAs-GaAs heterostructurawhich shows clearly the expected oscillations. The experimental results shown here are in agreement with previously published measurements of the gate current and the magnetocapacitance $[2,4,14]$ and can be explained by a 3D model of the heterostructure where the carrier density is assumed to have statistical inhomogeneities and the input DOS is Gaussian in accordance with the LOCA [13].

\section{References}

[1] E. Stahl, D. Weiss, G. Weimann, K. v. Klitzing, K. Ploog, J. Phys. C18 L783 (1985)

[2] D. Weiss, K. v. Klitzing, V. Mosser, Springer Series in Solid State Sciences 67: Two-Dimensional Systems: Physics and New Devices, Editors: G. Bauer, F. Kuchar and H. Heinrich, (Springer Verlag Berlin, Heidelberg, 204 (1986))

[3] M.G. Gavrilov, I.V. Kukushkin, JETP lett. 43, 103 (1986)

[4] V. Mosser, D. Weiss, K. v. Klitzing, K. Ploog, G. Weimann, Solid State Commun. 58, 5 (1985)

[5] T.P. Smith, B.B. Goldberg, P.J. Stiles and M. Heiblum, Phys. Rev. B32 2696 (1985)

[6] T.P. Smith, W.T. Wang, P.J. Stiles, M. Heiblum, Phys. Rev. B34 2696 (1986)

[7] E. Gornik, R. Lassnig, G. Strasser, H.L. Störmer, A.C. Gossard, W. Wiegmann, Phys. Rev. Lett. 54 , $1820(1985)$
[8] J.P. Eisenstein, H.L. Störmer, V. Narayanamurti, A.Y Cho,. A.C. Gossard, C.W. Tu, Phys. Rev. Lett. 55 , 875 (1985)

[9] V. Gudmundsson, R.R. Gerhardts, Phys. Rev. B to be published

[10] R.T. Zeller, F.F. Fang, B.B. Goldberg, S.L. Wright, P.J. Stiles, Phys. Rev. 33, 1529 (1986)

[11] R. R. Gerhardts and V. Gudmundsson, Phys. Rev. B34, 2999 (1986)

[12] T. Ando, Y. Uemura, J. Phys. Soc. Japan 36, 959 (1974);

T. Ando, J. Phys. Soc. Japan 37, 622 (1974)

[13] R.R. Gerhardts, Z. Physik B21, 275 (1975); 285 (1975); Surf. Sci. 58, 234 (1976)

[14] D. Weiss, K. v. Klitzing, Springer Series in Solid State Sciences Würzburg Conference 1986 , to be published 